\title{
Chromatin May Control How Droplets Form and Grow in Cells
}

\author{
In cell nuclei, molecular structures called chromatin may play a role in the \\ formation of droplets that are crucial for many cellular functions.
}

By Erika K. Carlson

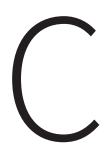
ells often contain droplets, which are involved in various cellular functions. The environments in which these droplets form are complicated-the cell nucleus, for example, is packed with a complex molecular structure called chromatin, which may affect how droplets merge, among other things. Now, Yaojun Zhang of Princeton University and colleagues have proposed a description of how chromatin affects droplet formation in cells [1]. The result highlights the crucial part played by the environment in the formation of cellular structures.

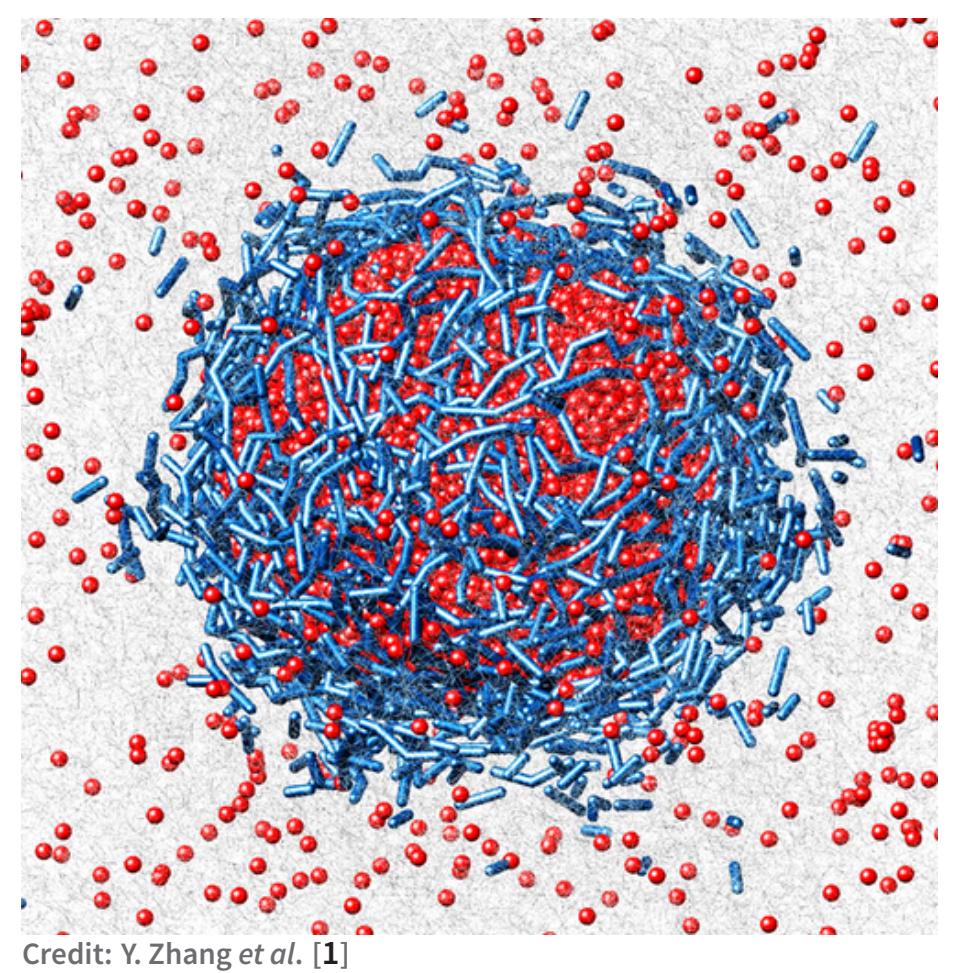

Credit: Y. Zhang et al. [1]
In their experiments, the team created liquid droplets of varying sizes inside the nucleus of a living cell. Conventional theoretical understanding of this situation, in which droplets of one immiscible liquid are dispersed in another, predicts that the larger droplets should swallow up the smaller ones and grow quickly. But Zhang and colleagues found that their droplets barely grew at all. They hypothesized that the presence of long, fiber-like structures-the chromatin-could be inhibiting the expected droplet growth. Previous work had suggested that networks of polymers can affect droplet formation in other scenarios.

The team then conducted molecular dynamics simulations to get a more detailed picture of how networks of crosslinked chromatin might affect droplet formation and growth. They found that crosslinked chromatin can act as a mechanical barrier to growth, with the fiber network pushing back against droplets that grow large enough to stretch it, limiting droplet size. The team suggests that this effect may be responsible for the suppression of large droplet growth that they observed in experiments. Considering factors such as pressure and chemical potential, the team found that the mechanical effects of chromatin might also affect the droplets' number, size, and placement.

Today, Physics published another cell story highlighting theoretical predictions that show that the number of molecular components in a cell directly determine the number of droplet types that can form (Synopsis: Predicting the Population of Self-Assembling Droplets in Cells).

Erika K. Carlson is a Corresponding Editor for Physics based in New York City. 
REFERENCES

1. Y. Zhang et al., "Mechanical frustration of phase separation in the cell nucleus by chromatin,” Phys. Rev. Lett. 126, 258102 (2021). 